\title{
Based on bioinformatics analysis, NF1, BTRC and MAPK14 are the potential biomarkers in the progression of osteoarthritis synovitis
}

\author{
Mingyi Yang \\ Xi'an Red Cross Hospital
}

Yani Su

Yan'an University

Yao Ma

Xi'an Red Cross Hospital

Yirixiati Aihaiti

Xi'an Red Cross Hospital

Peng Xu ( $\nabla$ sousou369@163.com )

Xi'an Red Cross Hospital https://orcid.org/0000-0003-2487-9163

\section{Research Article}

Keywords: osteoarthritis, genes, miRNA, synovitis

Posted Date: March 24th, 2021

DOI: https://doi.org/10.21203/rs.3.rs-349764/v1

License: (c) (i) This work is licensed under a Creative Commons Attribution 4.0 International License. Read Full License 


\section{Abstract}

Objective: To study the potential biomarkers and related pathways in osteoarthritis (OA) synovial lesions, and to provide theoretical basis and research directions for the pathogenesis and treatment of OA.

Methods: Download the microarray data sets GSE12021 and GSE82107 from Gene Expression Omnibus. GEO2R recognizes differentially expressed genes. Perform functional enrichment analysis of differentially expressed genes and construct protein-protein interaction network. Cytoscape performs module analysis and enrichment analysis of top-level modules. Further identify the Hub gene and perform functional enrichment analysis. TargetScan, miRDB and miRWalk three databases predict the target miRNAs of Hub gene and identify key miRNAs.

Results: Finally, 10 Hub genes and 17 key miRNAs related to the progression of OA synovitis were identified. NF1, BTRC and MAPK14 may play a vital role in OA synovial disease.

Conclusion: The Hub genes and key miRNAs discovered in this study may be potential biomarkers in the development of OA synovitis, and provide research methods and target basis for the pathogenesis and treatment of $O A$.

\section{Introduction}

Osteoarthritis $(\mathrm{OA})$ is a chronic degenerative joint disease that results from a variety of causes. It involves all tissues of the joint and is characterized by degeneration, destruction of articular cartilage and bone hyperplasia. During pathological changes, structural changes in the articular cartilage, synovium, subchondral bone and surrounding soft tissues (muscles, ligaments, etc.) can be observed. Although most of the research on $\mathrm{OA}$ in recent years has focused on the degradation of cartilage and changes in subchondral bone, synovitis, as one of the macroscopic structural changes of $O A$, has received increasing attention in the role of OA progression $(1,2)$. The articular microenvironment of low-grade inflammatory $\mathrm{OA}$ is caused by cartilage breakdown products that stimulate the release of proteolytic enzymes and the increase in FLS proinflammatory mediators, followed by immune cell infiltration and vascular hyperplasia, which ultimately leads to synovial inflammation $(1,2)$. This synovial change and overexpression of proinflammatory mediators can be observed in early OA. Synovitis is related to the symptoms and progression of $\mathrm{OA}$ and can be used as a potential target for OA treatment.

During recent decades, bioinformatics has become an important part of the entire life sciences development and has become the frontier of life science research. Its role in medical research is becoming increasingly important. In this study, we downloaded 2 datasets from Gene Expression Omnibus (GEO) for analysis and obtained differentially expressed genes (DEGs) of OA synovial tissue and normal synovial tissue. Subsequently, we conducted gene ontology (GO), Kyoto Encyclopedia of Genes and Genomes (KEGG) pathway enrichment analysis, and protein-protein interaction (PPI) network analysis and further explored the potential biological molecules related to the progression of OA synovitis to help us understand the pathogenesis of OA synovitis. 


\section{Material And Methods}

\section{Microarray data}

GEO (https://www.ncbi.nlm.nih.gov/geo) belongs to the NCBI ((https://www.ncbi.nlm.nih.gov/) of the National Institutes of Health. It is a public functional genomic database that stores high-throughput gene expression data, chips, and microarrays. We downloaded 2 gene expression datasets (GSE82107 and GSE12021) from GEO. The GSE82107 dataset contains 10 OA synovial tissue samples and 7 normal synovial tissue samples. GSE12021 contains 20 OA synovial tissue samples, 24 rheumatoid arthritis (RA) synovial tissue samples and 13 normal synovial tissue samples.

\section{Identification of DEGs}

GEO2R (http://www.ncbi.nlm.nih.gov/geo/geo2r) was used to screen DEGs between OA synovial tissue samples and normal synovial tissue samples. GEO2R is an interactive web tool that allows users to compare two or more samples in the GEO series to identify different genes expressed under different experimental conditions. After the two datasets were screened by GEO2R, they were standardized by $\mathrm{P}<0.05$ and $\log \mathrm{FC} \geq 1 / \log \mathrm{FC} \leq-1$, and finally, their respective $\mathrm{DEG}$ s were obtained.

\section{GO and KEGG enrichment analyses of DEGs}

DAVID (http://david.ncifcrf.gov, version 6.8) is a biological information database that integrates biological data and analysis tools. It provides comprehensive biological function annotation information for large-scale gene or protein lists to help users extract biological information from it (3). KEGG is a database that integrates genomic, chemical, and system function information. It is a computer simulation of a biological system. $\mathrm{GO}$ is a major bioinformatics tool for annotating genes and analyzing the biological processes of these genes (4), providing a series of terms to describe the characteristics of genes and gene products. Using the $\mathrm{GO}$ enrichment analysis method to annotate the biological process (BP), cellular component (CC) and molecular function (MF) of DEGs, with $\mathrm{P}<0.05$ as the statistical standard, when more than three enrichment items were found in each category, the first four enrichment items were selected according to the number of enriched genes. The KEGG analysis approach was used to determine gene clustering and related function paths, with $\mathrm{P}<0.05$ as the statistical standard, and the first five enrichment terms were selected according to the number of enriched genes. To analyze the function of DEGs, biological analyses were performed using the DAVID online database. $P<0.05$ was considered statistically significant. GraphPad was used to visualize the enrichment results and the corresponding number of DEGs.

\section{PPI network construction and module analysis}

Search Tool for the Retrieval of Interacting Genes (STRING; http://string-db.org) (version 11.0) is a system that searches for known proteins and predicts the interactions between proteins, which can predict the interaction gene network and provide a deeper scientific basis for understanding the mechanism of disease occurrence or development. Cytoscape (version 3.8.0) is a software program focused on open 
source network visualization and analysis. Its core is to provide a basic functional layout and query network and combine it into a visualization network based on basic data. Using STRING to predict the protein interaction relationship of DEGs, the interaction with a comprehensive score $>0.4$ was considered to be statistically significant, and Cytoscape was used to visualize the protein interaction network. Cytoscape's MCODE plug-in was used to obtain the top-level modules, and then the first 5 top-level modules were selected to be used in Cytoscape's ClueGO plug-in for GO and KEGG enrichment analysis.

\section{Hub gene selection}

Using Cytoscape's plug-in Cytohubba (Degree algorithm), the top 10 genes with the highest network connectivity in the PPI were selected as Hub genes, and the Hub gene interaction network was visualized and ranked.

\section{Hub gene analysis}

GO enrichment analysis of 10 Hub genes was performed using the BINGO plug-in of Cytoscape, with $\mathrm{P}<0.005$ as the statistical standard.

\section{Construction of the Hub gene-miRNA interaction network and prediction of key miRNAs}

To predict the target miRNAs of the Hub genes and to ensure the reliability of the prediction results, we used three databases, namely, TargetScan, miRDB and miRWalk, to take the intersection of the predicted results. Cytoscape is used to construct a Hub gene-miRNA interaction network, and miRNAs targeting two or more Hub genes are considered to be key miRNAs.

\section{Results}

\section{Identification of DEGs}

After the microarray results were standardized, GSE82107 identified 3404 DEGs, and GSE12021 identified 2710 DEGs. The two datasets overlapped and contained 626 genes, as shown in (Fig. 1). These 626 genes were either upregulated $(\log F C \geq 1)$ or downregulated $(\log F C \leq-1)$ in the two datasets GSE82107 and GSE12021. After discarding the genes that were inconsistent in the two datasets, 50 upregulated genes and 426 downregulated genes were identified, such that 476 DEGs were finally identified.

\section{GO and KEGG enrichment analyses of DEGs}

DEGs were analyzed for GO and KEGG enrichment using DAVID. GO analysis results showed that changes in BP of DEGs were significantly enriched in signal transduction, positive regulation of transcription from the RNA polymerase II promoter, positive regulation of GTPase activity and positive regulation of transcription, DNA-templated. Changes in the CC of DEGs were mainly enriched in the cytoplasm, nucleus, extracellular exosome and membrane. Changes in MF were mainly enriched in cadherin binding involved in cell-cell adhesion, protein serine/threonine kinase activity, actin binding and GTPase activator activity (Table 1). The number of DEGs in each GO enrichment area was different 
(Figure 2A). KEGG pathway analysis revealed that the DEGs were mainly enriched in the cAMP signaling pathway, Rap1 signaling pathway, platelet activation, signaling pathways regulating the pluripotency of stem cells and the Hippo signaling pathway (Table 2). The number of DEGs enriched in each signaling pathway was different (Figure 2B).

\section{PPI network construction and module analysis}

The PPI network of DEGs was constructed (Figure 3), and the most significant module was obtained using Cytoscape (Figure 4). The selection criteria were MCODE Cutoff $>5$, Degree Cutoff $=2$, Node Score Cutoff $=0.2$, Max Depth=100, and K-Core=2. The Cytoscape ClueGO plug-in was used to perform GO enrichment analysis on 5 modules (58 genes) (Figure 5A), with $\mathrm{P} \leq 0.05$ as the statistical standard. GO analysis results showed that changes in BP were significantly enriched in transmitter-gated ion channel activity, transmitter-gated channel activity and postsynaptic neurotransmitter receptor activity. Changes in $\mathrm{CC}$ were mainly enriched in the Golgi lumen, ionotropic glutamate receptor complex and postsynaptic density membrane. Changes in MF were mainly enriched in protein phosphorylated amino acid binding, frizzled binding and glutamate receptor activity (Figure 6A). KEGG enrichment analysis (Figure 5B) uses $P$ $\leq 0.05$ as the statistical standard, and the results were mainly enriched in long-term potentiation, nicotine addiction, signaling pathways regulating the pluripotency of stem cells, melanogenesis and basal cell carcinoma (Figure 6B).

\section{Hub gene selection}

Screened by Cytoscape, 10 Hub genes are displayed in the PPI network (Figure 7A), and 10 Hub genes were connected to each other (Figure 7B). The ranking of the Hub genes in the Cytoscape Cytohubba plug-in (Degree algorithm) from high to low is SRC, SOCS3, MAPK14, GRIA1, PTPN11, BTRC, GRIN1, UBE3A, NF1, and STAT1 (Table 3).

\section{Hub gene analysis}

Using Cytoscape to perform GO enrichment analysis of the Hub genes (Figure 8), with the GO enrichment result $\mathrm{P}<0.01$ as the statistical standard, the Hub genes were significantly enriched in the regulation of cellular processes, cytoplasm, biological regulation, multicellular organismal process, signal transduction, response to chemical stimulus, intracellular protein kinase cascade and cell surface receptor linked signaling pathway (Table 4).

\section{miRNA mining and Hub gene-miRNA interaction network analysis}

Three databases, namely, TargetScan, miRDB and miRWalk, were used to predict the target miRNAs of the Hub genes, and the prediction result was visualized by Cytoscape. The interaction network of the Hub genes and miRNAs was constructed (Figure 9). miRNAs targeting two Hub genes at the same time were defined as key miRNAs, and a total of 17 key miRNAs were obtained (Table 5). 


\section{Discussion}

$\mathrm{OA}$ is a chronic degenerative disease characterized by articular cartilage degeneration and bone hyperplasia. The pathological features are articular cartilage degeneration and destruction, subchondral bone sclerosis or cystic degeneration, joint edge bone hyperplasia, synovial lesions, joint capsule contracture, relaxation or contracture of ligaments, muscle atrophy, etc. At present, the etiology of OA is not clear. Existing studies have shown that age, sex, family history, obesity, trauma, and increased joint weight are all related to the occurrence of OA (5). Cartilage, synovium, and subchondral bone are thought to be involved in the production of inflammatory cytokines, but the degree of action of each component is unclear. Synovitis is one of the macroscopic structural changes of OA, affecting joint integrity (6). Knee OA synovitis is considered to be a secondary reaction caused by certain stimulation of residual articular cartilage and debris and is the main cause of joint swelling and pain (7). The cause of OA synovitis is unknown, and stimulation of articular cartilage residues and debris is only one of the mechanisms that cause clinical symptoms of knee OA. In the late stage of OA, the synovial membrane of the joint cavity is stimulated for a long time and exhibits villous hyperplasia, and the surface of the articular cartilage erodes, thereby destroying the cartilage and bone and accelerating the progression of OA (8). Studies have shown that fibroblast-like synovial cells(FLS) transformation and synovitis contribute to the occurrence of inflammatory arthritis $(9,10)$. The transformed FLSs are aggressive and cause persistent synovitis. They can proliferate rapidly and are highly migratory, attach to articular cartilage and produce matrix-degrading enzymes to degrade and invade cartilage (9). In RA and OA synovitis, the FLS invasion behavior may be different in distribution. The synovial inflammation of RA is diffusely distributed, while the synovitis of OA is usually patchy and confined to the area adjacent to the cartilage injury (6). Affected by the distribution of joint synovial inflammatory tissue, the pathological changes in OA may also be different (11). The severity of OA synovitis may affect the expression levels of cell adhesion- and cell movement-related genes in FLSs (12). Synovitis is one of the macroscopic structural changes of OA. Studying the signaling molecules related to the abnormal changes in OA synovial cells has important theoretical and clinical significance for an in-depth understanding of the occurrence and development of $\mathrm{OA}$ and the role of synovial cells in $\mathrm{OA}$.

Microarray and sequencing technologies provide the expression levels of thousands of genes in humans and have been widely used to predict potential therapeutic targets for diseases. The purpose of this study was to determine the potential biomarkers related to the OA synovial inflammation process through microarray and bioinformatics analysis to obtain a deeper understanding of the etiology and pathogenesis of OA synovitis. In this study, two datasets, GSE12021 and GSE82107, were downloaded from GEO. A total of 476 DEGs were identified, including 50 upregulated genes and 426 downregulated genes. Enrichment analysis of DEGs showed that changes in BP were significantly enriched in signal transduction and positive regulation of transcription from the RNA polymerase II promoter; changes in CC were mainly enriched in cytoplasm and nucleus; and changes in MF were mainly enriched in cadherin binding involved in cell-cell adhesion and protein serine/threonine kinase activity. The PPI network of DEGs has a total of 9 top-level modules. The top 5 top-level modules interact closely and contain a total of 58 genes. Compared with other genes, these 58 genes may be potential biomarkers and have potential 
value in the pathogenesis of OA synovitis. The 10 genes with the highest network connectivity in PPI were defined. We found that 6 of these 10 Hub genes occurred among the 58 genes in the top 5 modules, namely, SOCS3, GRIA1, PTPN11, BTRC, GRIN1 and UBE3A, which shows that these 6 genes are most likely potential biomarkers in the pathogenesis of OA synovitis. The further enrichment of 10 Hub genes and comprehensive analysis showed that most of these 10 Hub genes were enriched in the regulation of cellular processes, multicellular organismal process and signal transduction, which may play an important role in the occurrence and development of OA synovitis.

Immediately afterwards, we conducted deeper miRNA mining of these 10 Hub genes, defined miRNAs targeting more than two Hub genes as key miRNAs, and finally obtained 17 key miRNAs related to the progression of OA synovitis. Through the mutual targeting relationship between Hub genes and key miRNAs, we found that most of these 17 key miRNAs target the three Hub genes NF1, BTRC and MAPK14. We believe that NF1, BTRC and MAPK14 are potential biomarkers in the progression of OA synovitis and play an important role in the etiology and pathogenesis of OA.

NF1 is a major tumor suppressor gene located on chromosome 17q11.2. NF1 encodes a RAS (rat sarcoma)-GAP (GTPase activating protein) known as neurofibromin. Mutations in this gene are associated with neurofibromatosis type 1 (13), juvenile myeloid leukemia, and Watson syndrome. The mRNA for this gene is subject to RNA editing (CGA>UGA->Arg1306Term), resulting in premature translation termination. Studies have found that the NF1 gene is not only associated with tumor syndrome and melanoma (14) but also that NF1 deletion plays a leading role in the tumorigenesis of neurofibromatosis type 1 (15). Studies have also reported that neurofibrillin regulates the expression of FGF23(fibroblast growth factor 23) through the PI3K(phosphatidylinositol 3-kinase) pathway and plays an important role in bone cells. Bone cell NF1 loss leads to a significant increase in serum FGF23, and mineralization defects lead to bone hyperplasia, which leads to a significant decrease in bone mechanical strength (16). However, there is no report on the research of NF1 and OA. In this study, we found that NF1 may be a potential biological molecule of OA synovitis. The role of NF1 in bone cells has been studied in the past. The correlation between NF1 and OA is of relatively great research value.

BTRC encodes a $\beta$-transducer protein ( $\beta$-TrCP) containing a repeat sequence. It is a member of the E3 ubiquitin ligase family containing F-box and WD40 repeat sequences and is located on chromosome $10 q 24$ q25. $(17,18)$. Over the years, studies have found that BTRC is involved in the development of various diseases. EBV-miR-BART10-3p promotes epithelial-mesenchymal transition by targeting BTRC and leads to metastasis of nasopharyngeal carcinoma; TSPAN15(tetraspanin 15) and BTRC promote esophageal squamous cell carcinoma metastasis by activating NF-KB signaling (19); gene mutations of WNT2B(Wnt family member 2B) and BTRC can predict the survival of melanoma (20); deletion of BTRC can cause abnormal development of mouse retina (21). However, the role of BTRC in the progression of OA has not been reported. This study found that BTRC is expressed at low levels in the occurrence and development of OA synovitis, which may be a potential factor for the progression of OA synovitis. 
MAPK14 is a member of the MAP(methionine aminopeptidase) kinase family. As an integration point of various biochemical signals, MAP kinase is involved in various processes, such as cell proliferation, differentiation, transcriptional regulation and development. The substrates of this kinase include the transcription regulators ATF2(activating transcription factor 2), MEF2C(myocyte enhancer factor 2C) and MAX(MYC associated factor $X$ ); the cell cycle regulator CDC25B(cell division cycle 25B); and the tumor suppressor $\mathrm{p} 53$, suggesting that this kinase plays a role in stress-related transcription and cell cycle regulation and the genotoxic stress response. Studies have shown that MAPK14 is highly expressed in many tumors, such as breast cancer, ovarian serous adenocarcinoma, and gastric cancer, and promotes the occurrence, invasion and migration of tumor cells (22-24). MAPK14 is a candidate gene for genetic susceptibility to diabetic foot ulcers (25). Downregulation of MAPK14 can inhibit the proliferation and migration of clear cell renal cell carcinoma by downregulating the expression of CDC25B (26). MicroRNA124 can inhibit the activation of the MAPK signaling pathway by downregulating MAPK14, thereby reducing lung injury in septic shock mice (27). In this study, we found that MAPK14 may play an important role in OA synovitis, and the study of its specific mechanism has certain value for the diagnosis and treatment of $\mathrm{OA}$.

As with previous bioinformatics analysis research, this research has several limitations. First and foremost, the sample size of this study is relatively small, and the sampling method does not eliminate the effects of sex, comorbidities and the use of certain drugs that can alter the expression of OA genes. Second, the Hub genes, key miRNAs and related pathways obtained from the research have not been confirmed by other functional studies, but this will be an area for further research. Since many genes found in this study were not previously associated with $O A$, further studies are needed to verify the expression of these genes in OA synovium and healthy control synovial tissue samples.

\section{Conclusion}

In this study, we applied a series of comprehensive bioinformatics analyses to screen the key genes and pathways related to the occurrence and development of OA synovitis. Finally, 10 hub genes and 17 key miRNAs were identified, which may be potential biomarkers in the progression of OA synovitis. Through the mutual targeting relationship between the Hub genes and key miRNAs, we found that the three Hub genes NF1, BTRC and MAPK14 are in an important position and play a central role in the etiology and pathogenesis of OA synovitis. These potential biomarkers may provide a basis for the study of therapeutic targets for OA synovitis, and further molecular biology experiments are needed to analyze and verify these potential markers and pathways to study their potential role in future diagnosis, prognosis and treatment.

\section{List Of Abbreviations}

OA: osteoarthritis

GEO: Gene Expression Omnibus 
DEGs: differentially expressed genes

GO: gene ontology

KEGG: Kyoto Encyclopedia of Genes and Genomes

PPI: protein-protein interaction network

RA: rheumatoid arthritis

BP: biological process

CC: cellular component

MF: molecular function

STRING: Search Tool for the Retrieval of Interacting Genes

FLS: fibroblast-like synovial cells

RAS: rat sarcoma

GAP: GTPase activating protein

FGF23: fibroblast growth factor 23

PI3K: phosphatidylinositol 3-kinase

TSPAN15: tetraspanin 15)

WNT2B: Wnt family member 2B

MAP: methionine aminopeptidase

ATF2: activating transcription factor 2

MEF2C: myocyte enhancer factor 2C

MAX: MYC associated factor $X$

CDC25B: cell division cycle 25B

\section{Declarations}

Acknowledgements: We thank Mr Yongsong Cai and Mr Ke Xu for their support in bioinformatics and language. 
Publishing consent: So the authors agree that the article will be published in your journal.

Funding: This work was financially supported by the National Natural Science Foundation of China (No. 81772410, 81601877ه खChina Postdoctoral Science Foundation(No. 2020M673454)

Conflict of interest reputation: There is no conflict of interest between all authors of this article.

Data availability claims: The data used in this article comes from Gene Expression Omnibus and has high availability.

Authors' contributions reputation: Peng Xu and Mingyi Yang designed the study. Yani Su, Yao Ma and Ti Xia analyzed the microarray datasets and interpreted the results. Mingyi Yang downloaded the gene expression profile from the Gene Expression Omnibus. Mingyi Yang and Yani Su wrote and edited the manuscript. Peng Xu provided the foundation and support, All authors read and approved the final manuscript.

\section{References}

1. Mathiessen A, Conaghan PG. Synovitis in osteoarthritis: current understanding with therapeutic implications. Arthritis Res Ther. 2017; 19: 18.

2. Attur M, Samuels J, Krasnokutsky S, et al. Targeting the synovial tissue for treating osteoarthritis (OA): where is the evidence? Best Pract Res Clin Rheumatol. 2010; 24: 71-9.

3. Huang DW, Sherman BT, Tan Q, et al. The DAVID Gene Functional Classification Tool: a novel biological module-centric algorithm to functionally analyze large gene lists. Genome Biol. 2007; 8: R183.

4. Ashburner M, Ball CA, Blake JA, et al. Gene ontology: tool for the unification of biology. The Gene Ontology Consortium. Nat Genet. 2000; 25: 25-9.

5. Allen KD, Golightly YM. State of the evidence. Curr Opin Rheumatol. 2015; 27: 276-83.

6. Sellam J, Berenbaum $F$. The role of synovitis in pathophysiology and clinical symptoms of osteoarthritis. Nat Rev Rheumatol. 2010; 6: 625-35.

7. Benito MJ, Veale DJ, FitzGerald 0 , et al. Synovial tissue inflammation in early and late osteoarthritis. Annals of the rheumatic diseases. 2005; 64: 1263-7.

8. O'Neill TW, Parkes MJ, Maricar N, et al. Synovial tissue volume: a treatment target in knee osteoarthritis (OA). Annals of the rheumatic diseases. 2016; 75: 84-90.

9. Bhattaram P, Chandrasekharan U. The joint synovium: A critical determinant of articular cartilage fate in inflammatory joint diseases. Semin Cell Dev Biol. 2017; 62: 86-93.

10. Bottini N, Firestein GS. Duality of fibroblast-like synoviocytes in RA: passive responders and imprinted aggressors. Nat Rev Rheumatol. 2013; 9: 24-33.

11. Shibakawa A, Aoki H, Masuko-Hongo K, et al. Presence of pannus-like tissue on osteoarthritic cartilage and its histological character. Osteoarthritis Cartilage. 2003; 11: 133-40. 
12. Scanzello CR. Chemokines and inflammation in osteoarthritis: Insights from patients and animal models. Journal of orthopaedic research : official publication of the Orthopaedic Research Society. 2017; 35: 735-39.

13. Assunto A, Ferrara U, De Luca A, et al. Isoform-specific NF1 mRNA levels correlate with disease severity in Neurofibromatosis type 1. Orphanet J Rare Dis. 2019; 14: 261.

14. Kiuru M, Busam KJ. The NF1 gene in tumor syndromes and melanoma. Lab Invest. 2017; 97: 146-57.

15. Pemov A, Li H, Patidar R, et al. The primacy of NF1 loss as the driver of tumorigenesis in neurofibromatosis type 1-associated plexiform neurofibromas. Oncogene. 2017; 36: 3168-77.

16. Kamiya N, Yamaguchi R, Aruwajoye O, et al. Targeted Disruption of NF1 in Osteocytes Increases FGF23 and Osteoid With Osteomalacia-like Bone Phenotype. J Bone Miner Res. 2017; 32: 1716-26.

17. Kipreos ET, Pagano M. The F-box protein family. Genome Biology 2000; 1: 3002.1-02.7.

18. Jin J, Cardozo T, Lovering RC, et al. Systematic analysis and nomenclature of mammalian F-box proteins. Genes Dev. 2004; 18: 2573-80.

19. Zhang B, Zhang Z, Li L, et al. TSPAN15 interacts with BTRC to promote oesophageal squamous cell carcinoma metastasis via activating NF-kappaB signaling. Nat Commun. 2018; 9: 1423.

20. Shi Q, Liu H, Han P, et al. Genetic Variants in WNT2B and BTRC Predict Melanoma Survival. J Invest Dermatol. 2017; 137: 1749-56.

21. Baguma-Nibasheka M, Kablar B. Abnormal retinal development in the Btrc null mouse. Dev Dyn. 2009; 238: 2680-7.

22. Chiacchiera F, Simone C. Signal-dependent regulation of gene expression as a target for cancer treatment: inhibiting p38alpha in colorectal tumors. Cancer Lett. 2008; 265: 16-26.

23. Song WJ, Dong Y, Luo C, et al. p38MAPK family isoform p38alpha and activating transcription factor 2 are associated with the malignant phenotypes and poor prognosis of patients with ovarian adenocarcinoma. Pathol Res Pract. 2017; 213: 1282-88.

24. Zhao L, Jiang L, He L, et al. Identification of a novel cell cycle-related gene signature predicting survival in patients with gastric cancer. J Cell Physiol. 2019; 234: 6350-60.

25. Laimer M. MAPK14 as candidate for genetic susceptibility to diabetic foot ulcer. Br J Dermatol. 2017; 177: $1482-83$.

26. Liu J, Yu X, Yu H, et al. Knockdown of MAPK14 inhibits the proliferation and migration of clear cell renal cell carcinoma by downregulating the expression of CDC25B. Cancer Med. 2020; 9: 1183-95.

27. Pan W, Wei N, Xu W, et al. MicroRNA-124 alleviates the lung injury in mice with septic shock through inhibiting the activation of the MAPK signaling pathway by downregulating MAPK14. Int Immunopharmacol. 2019; 76: 105835.

\section{Tables}

Table1. GO enrichment analysis of DEGs in OA samples. 


\begin{tabular}{cccc}
\hline Term & Description & $\begin{array}{c}\text { Count in } \\
\text { gene set }\end{array}$ & P-value \\
\hline GO:0007165 & $\begin{array}{c}\text { signal transduction } \\
\text { GO:0045944 }\end{array}$ & 44 & $7.62 \mathrm{E}-04$ \\
& positive regulation of transcription from RNA & 36 & 0.004307 \\
gO:0043547 & $\begin{array}{c}\text { polymerase II promoter } \\
\text { positive regulation of GTPase activity }\end{array}$ & 21 & 0.02933 \\
GO:0045893 & positive regulation of transcription, DNA- & 20 & 0.022799 \\
templated & 158 & $7.75 \mathrm{E}-07$ \\
GO:0005737 & cytoplasm & 137 & 0.019337 \\
GO:0005634 & nucleus & 74 & 0.049351 \\
GO:0016062 & extracellular exosome & 70 & $8.42 \mathrm{E}-04$ \\
GO:0098641 & membrane & 16 & 0.002074 \\
GO:0004674 & cadherin binding involved in cell-cell adhesion & 16 & 0.020479 \\
GO:0003779 & protein serine/threonine kinase activity & 12 & 0.045307 \\
GO:0005096 & actin binding & 12 & 0.046391 \\
\hline GO, Gene Ontology; DEGs, differentially expressed genes; OA, Osteoarthritis. \\
\hline \multicolumn{4}{l}{} \\
\hline
\end{tabular}

Table 2. KEGG pathway enrichment analysis of DEGs in OA samples.

\begin{tabular}{|c|c|c|c|c|}
\hline Term & $\overline{\text { Description }}$ & Count & $\overline{\text { P-value }}$ & Genes \\
\hline hsa04024 & cAMP signaling pathway & 10 & 0.035686 & $\begin{array}{c}\text { ADCY1, SSTR2, PTGER3, } \\
\text { GRIA1, PDE4A, GRIN1, } \\
\text { GNAS, AFDN PDE4C, AKT3 }\end{array}$ \\
\hline hsa04015 & Rap1 signaling pathway & 10 & 0.048847 & $\begin{array}{c}\text { ADCY1, FLT1, MAPK14, } \\
\text { GRIN1, EFNA5, GNAS, } \\
\text { AFDN, RAPGEF1, AKT3, SRC }\end{array}$ \\
\hline hsa04611 & Platelet activation & 9 & 0.00919 & $\begin{array}{c}\text { GNA13, ADCY1, MAPK14, } \\
\text { GUCY1A2, } \\
\text { GNAS, COL1A1, ARHGEF12, } \\
\text { AKT3, SRC }\end{array}$ \\
\hline hsa04550 & $\begin{array}{l}\text { Signaling pathways } \\
\text { regulating pluripotency of } \\
\text { stem cells }\end{array}$ & 9 & 0.013986 & $\begin{array}{c}\text { ACVR1B, WNT10A, WNT7B, } \\
\text { NANOG, WNT5B, RIF1, } \\
\text { ESRRB, MAPK14, AKT3 }\end{array}$ \\
\hline hsa04390 & Hippo signaling pathway & 9 & 0.021139 & $\begin{array}{l}\text { WNT10A, WNT7B, TCF7, } \\
\text { WNT5B, NF2, BTRC, WWC1, } \\
\text { PATJ, DLG1 }\end{array}$ \\
\hline $\begin{array}{l}\mathrm{EGG}, \mathrm{KJ} \\
\text { A, Ostec }\end{array}$ & $\begin{array}{l}\text { Encyclopedia of Genes } \\
\text { ritis }\end{array}$ & & & \\
\hline
\end{tabular}

Table3ロHub gene ranking. 


\begin{tabular}{|c|c|c|c|}
\hline Gene & \begin{tabular}{|c} 
Details \\
\end{tabular} & Score & Species \\
\hline SRC & SRC proto-oncogene, non-receptor tyrosine kinase(SRC) & 41 & Homo \\
\hline SOCS3 & suppressor of cytokine signaling 3(SOCS3) & 21 & $\begin{array}{c}\text { sapiens } \\
\text { Homo }\end{array}$ \\
\hline MAPK14 & mitogen-activated protein kinase 14(MAPK14) & 20 & $\begin{array}{c}\text { Sapiens } \\
\text { Homo }\end{array}$ \\
\hline GRIA1 & glutamate ionotropic receptor AMPA type subunit & 19 & Homo \\
\hline PTPN11 & $\begin{array}{l}\text { protein tyrosine phosphatase, non-receptor type } \\
11(\mathrm{PTPN} 11)\end{array}$ & 19 & $\begin{array}{l}\text { Homo } \\
\text { sapiens }\end{array}$ \\
\hline BTRC & $\begin{array}{c}\text { beta-transducin repeat containing E3 ubiquitin protein } \\
\text { ligase(BTRC) }\end{array}$ & 17 & $\begin{array}{l}\text { Homo } \\
\text { sapiens }\end{array}$ \\
\hline GRIN1 & $\begin{array}{l}\text { glutamate ionotropic receptor NMDA type subunit } \\
\text { 1(GRIN1) }\end{array}$ & 17 & $\begin{array}{l}\text { Homo } \\
\text { sapiens }\end{array}$ \\
\hline UBE3A & ubiquitin protein ligase E3A(UBE3A) & 17 & Homo \\
\hline NF1 & neurofibromin 1 (NF1) & 17 & Homo \\
\hline STAT1 & signal transducer and activator of transcription 1(STAT1) & 16 & $\begin{array}{l}\text { Homo } \\
\text { sapiens }\end{array}$ \\
\hline
\end{tabular}

Table4. GO enrichment analysis of Hub gene in OA samples.

\begin{tabular}{|c|c|c|c|c|}
\hline GO-ID & Description & Count & $\begin{array}{c}\text { p- } \\
\text { value }\end{array}$ & Gene \\
\hline 50794 & $\begin{array}{l}\text { regulation of cellular } \\
\text { process }\end{array}$ & 10 & $\begin{array}{l}2.73 \mathrm{E}- \\
05\end{array}$ & GRIA1, SOCS3, SRC, STAT1, NF1, \\
\hline 5737 & cytoplasm & 10 & $\begin{array}{l}2.15 \mathrm{E}- \\
04\end{array}$ & GRIA1, SOCS3, SRC, STAT1, NF1, \\
\hline 65007 & biological regulation & 10 & $\begin{array}{l}8.17 \mathrm{E}- \\
05\end{array}$ & $\begin{array}{l}\text { UBE3A, MAPK14, BTRC, GRIN1 } \\
\text { GRIA1, SOCS3, SRC, STAT1, NF1, } \\
\text { PTPN11, }\end{array}$ \\
\hline 32501 & $\begin{array}{l}\text { multicellular organismal } \\
\text { process }\end{array}$ & 9 & $\begin{array}{l}1.12 \mathrm{E}- \\
03\end{array}$ & $\begin{array}{l}\text { UBE3A, MAPK14, BTRC, GRIN1 } \\
\text { GRIA1, SOCS3, SRC, STAT1, NF1, } \\
\text { PTPN11, UBE3A, BTRC, GRIN1 }\end{array}$ \\
\hline 7165 & signal transduction & 8 & $\begin{array}{l}5.63 \mathrm{E}- \\
07\end{array}$ & $\begin{array}{l}\text { GRIA1, SOCS3, SRC, STAT1, NF1, } \\
\text { PTPN11, MAPK14, BTRC }\end{array}$ \\
\hline 42221 & $\begin{array}{l}\text { response to chemical } \\
\text { stimulus }\end{array}$ & 7 & $\begin{array}{l}2.43 \mathrm{E}- \\
06\end{array}$ & $\begin{array}{l}\text { SOCS3, SRC, STAT1, NF1, } \\
\text { PTPN11, MAP14, GRIN1 }\end{array}$ \\
\hline 7243 & $\begin{array}{l}\text { intracellular protein kinase } \\
\text { cascade }\end{array}$ & 6 & $\begin{array}{l}1.38 \mathrm{E}- \\
08\end{array}$ & $\begin{array}{c}\text { SOCS3, SRC, STAT1, NF1, } \\
\text { PTPN11, MAPK14 }\end{array}$ \\
\hline 7166 & $\begin{array}{c}\text { cell surface receptor linked } \\
\text { signaling pathway }\end{array}$ & 6 & $\begin{array}{l}2.24 \mathrm{E}- \\
05\end{array}$ & $\begin{array}{c}\text { SRC, STAT1, PTPN11, MAPK14, } \\
\text { BTRC, GRIN1 }\end{array}$ \\
\hline
\end{tabular}

Table5. miRNAs and its target genes 


\begin{tabular}{|c|c|c|}
\hline miRNA & Genes targeted by miRNA & Gene count \\
\hline hsa-miR-103a-3p & BTRCaNF1 & 2 \\
\hline hsa-miR-107 & BTRC $\square$ NF1 & 2 \\
\hline hsa-miR-15a-5p & BTRC $\square$ NF1 & 2 \\
\hline hsa-miR-15b-5p & BTRC $\square$ NF1 & 2 \\
\hline hsa-miR-195-5p & BTRC $\square$ NF1 & 2 \\
\hline hsa-miR-424-5p & BTRC $\square$ NF1 & 2 \\
\hline hsa-miR-497-5p & BTRC $\square$ NF1 & 2 \\
\hline hsa-miR-6838-5p & BTRC $\square$ NF1 & 2 \\
\hline hsa-miR-1271-5p & GRIA1ロNF1 & 2 \\
\hline hsa-miR-96-5p & GRIA1ロNF1 & 2 \\
\hline hsa-miR-128-3p & MAPK14ロNF1 & 2 \\
\hline hsa-miR-216a-3p & MAPK14ロNF1 & 2 \\
\hline hsa-miR-27a-3p & MAPK14ロNF1 & 2 \\
\hline hsa-miR-27b-3p & MAPK14ロNF1 & 2 \\
\hline hsa-miR-3681-3p & MAPK14ロNF1 & 2 \\
\hline hsa-miR-141-3p & SRC $\square$ UBE3A & 2 \\
\hline hsa-miR-200a-3p & SRCIUBE 3A & 2 \\
\hline
\end{tabular}

\section{Figures}




\section{GSE12021}

\section{4}

\section{6}

2778

\section{GSE82107}

Figure 1

Datasets GSE82107 and GSE12021 select P<0.05, logFC $\geq 1 / \log F C \leq-1$ DEGs. Two datasets showed overlap of 626 genes. 

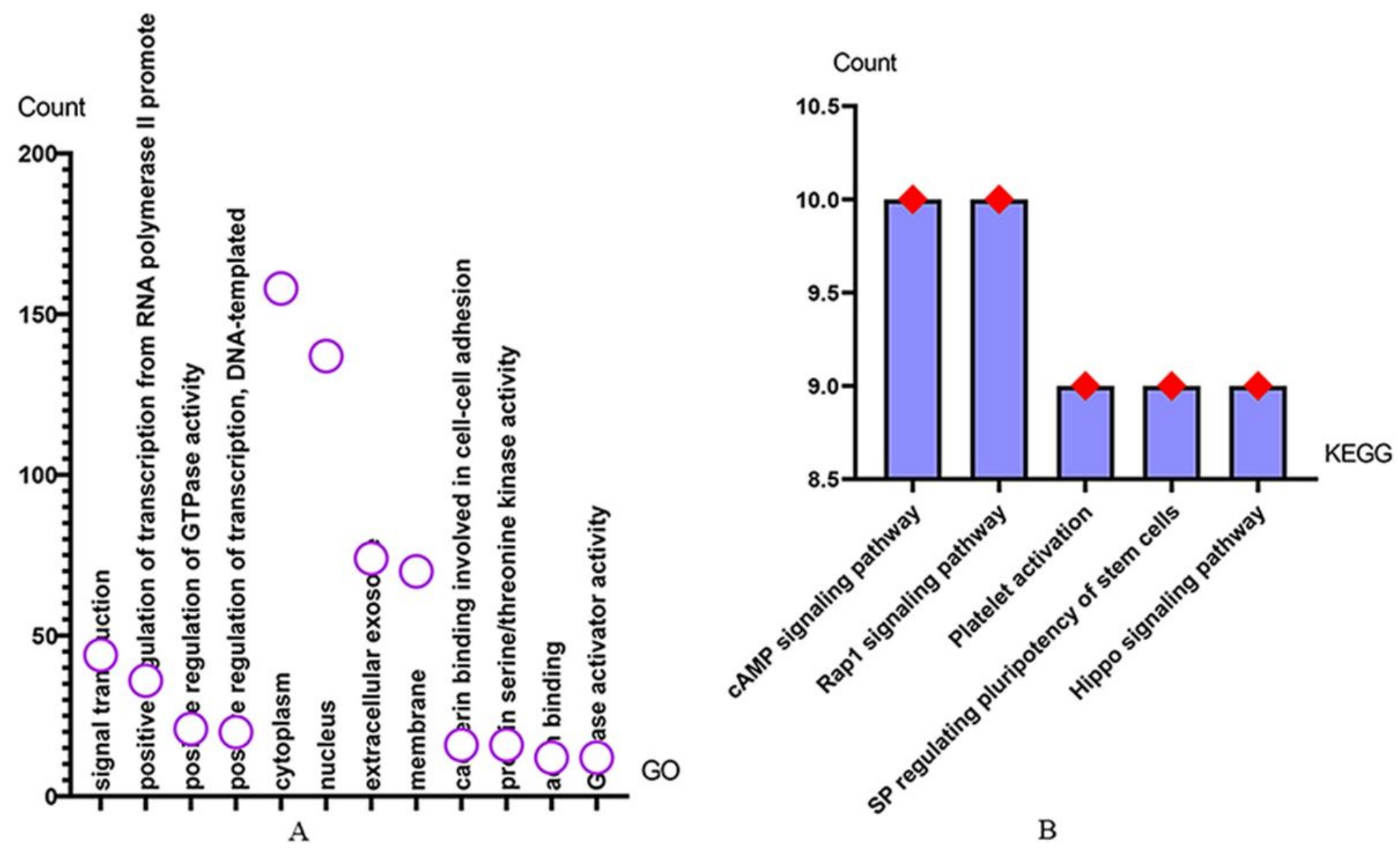

Figure 2

Enrichment results of DEGs for GO and KEGG. A: GO enrichment ,x-coordinate: $G 0$ enrichment results, $y$ coordinate: the number of DEGs enriched on this result. B: KEGG enrichment , $x$-coordinate: the enrichment results of KEGG pathway, $y$-coordinate: the number of DEGs enriched in this pathway. 


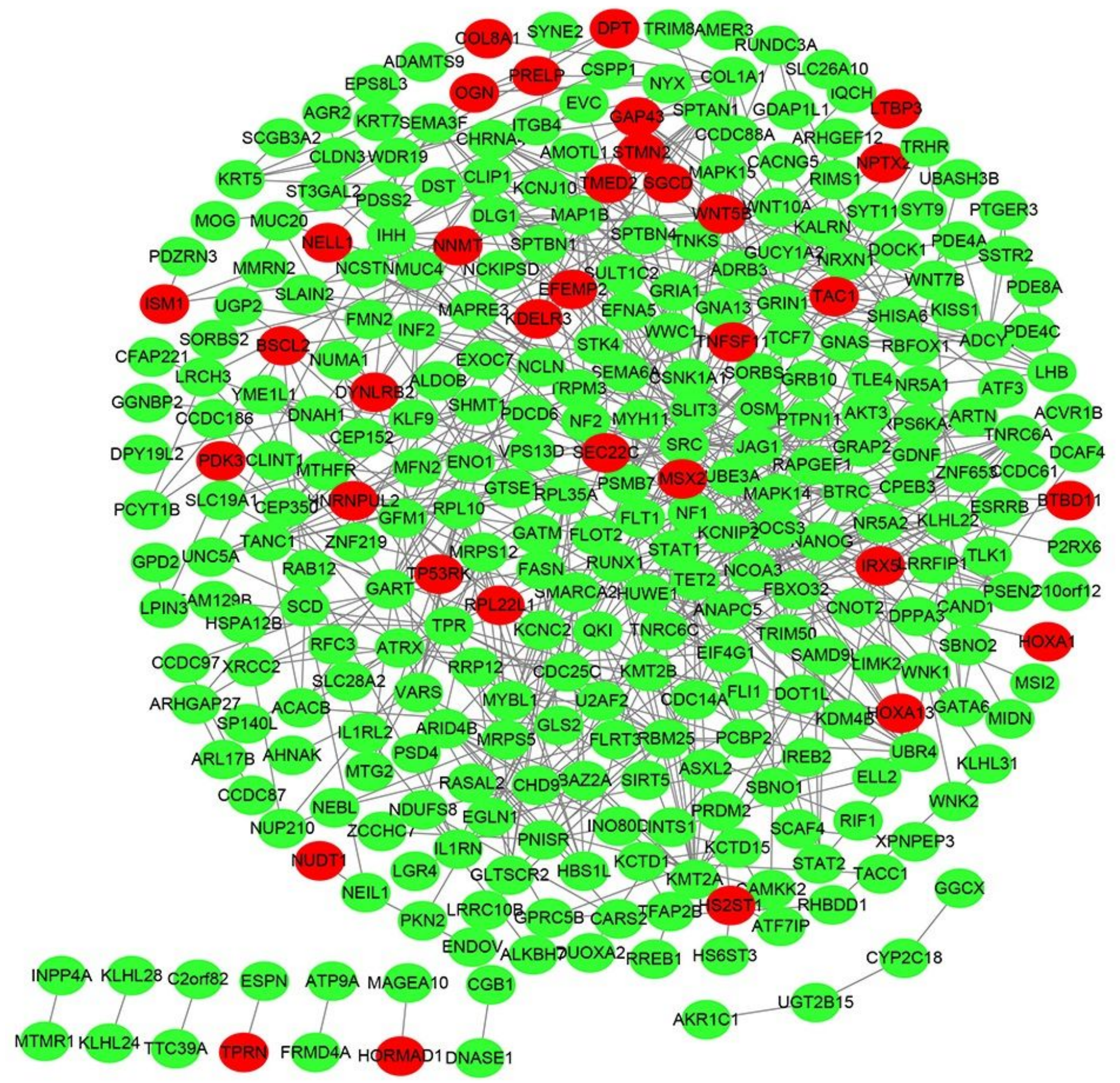

\section{Figure 3}

The PPI network of DEGs was constructed using Cytoscape. . The circles represent differential genes, red for up-regulated genes and green for down-regulated genes. 


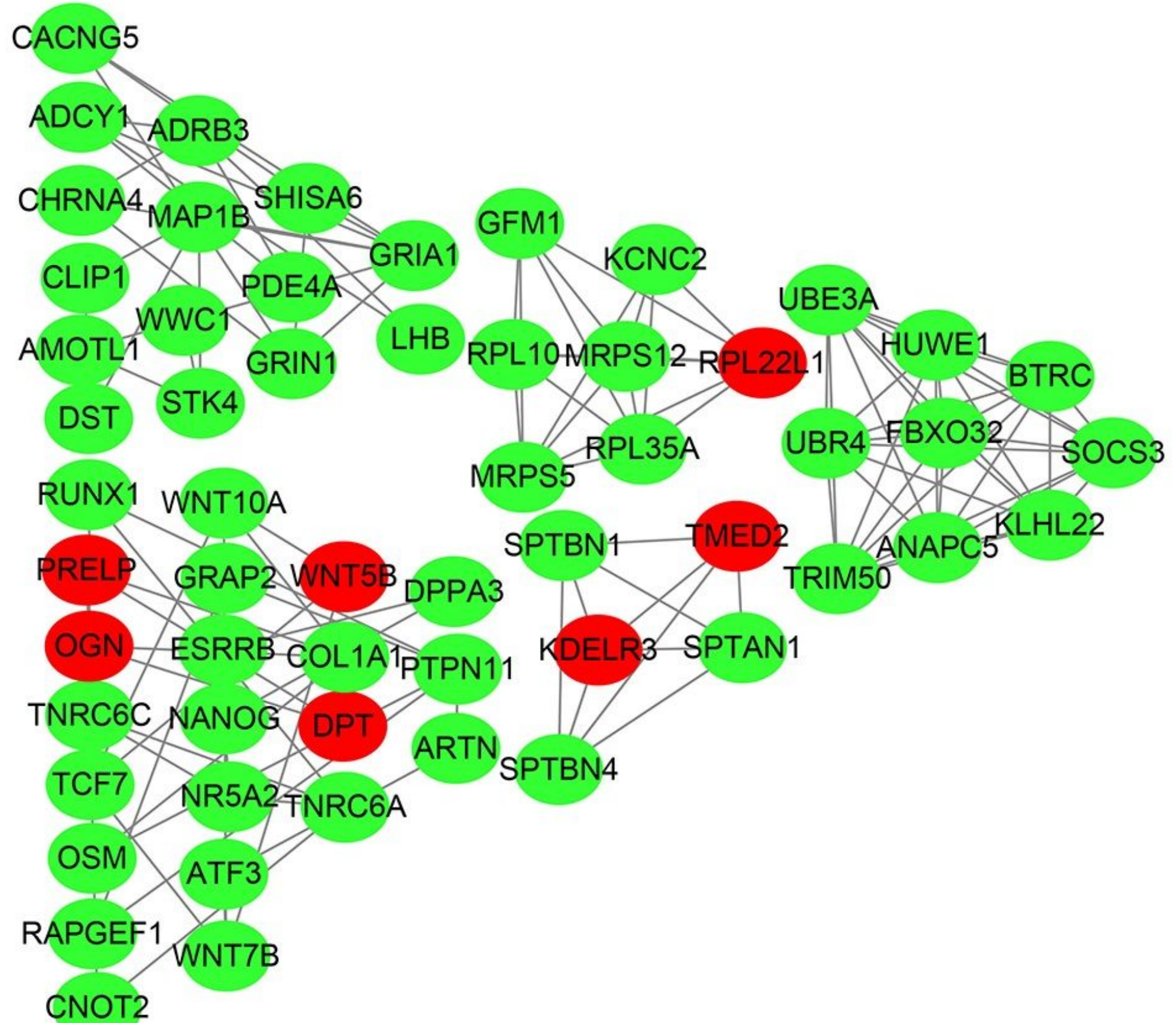

Figure 4

Rhomboid is the differential gene in 5 top modules, red is the upregulated gene, green is the downregulated gene. It is divided into 5 modules, each of which is an independent whole. 

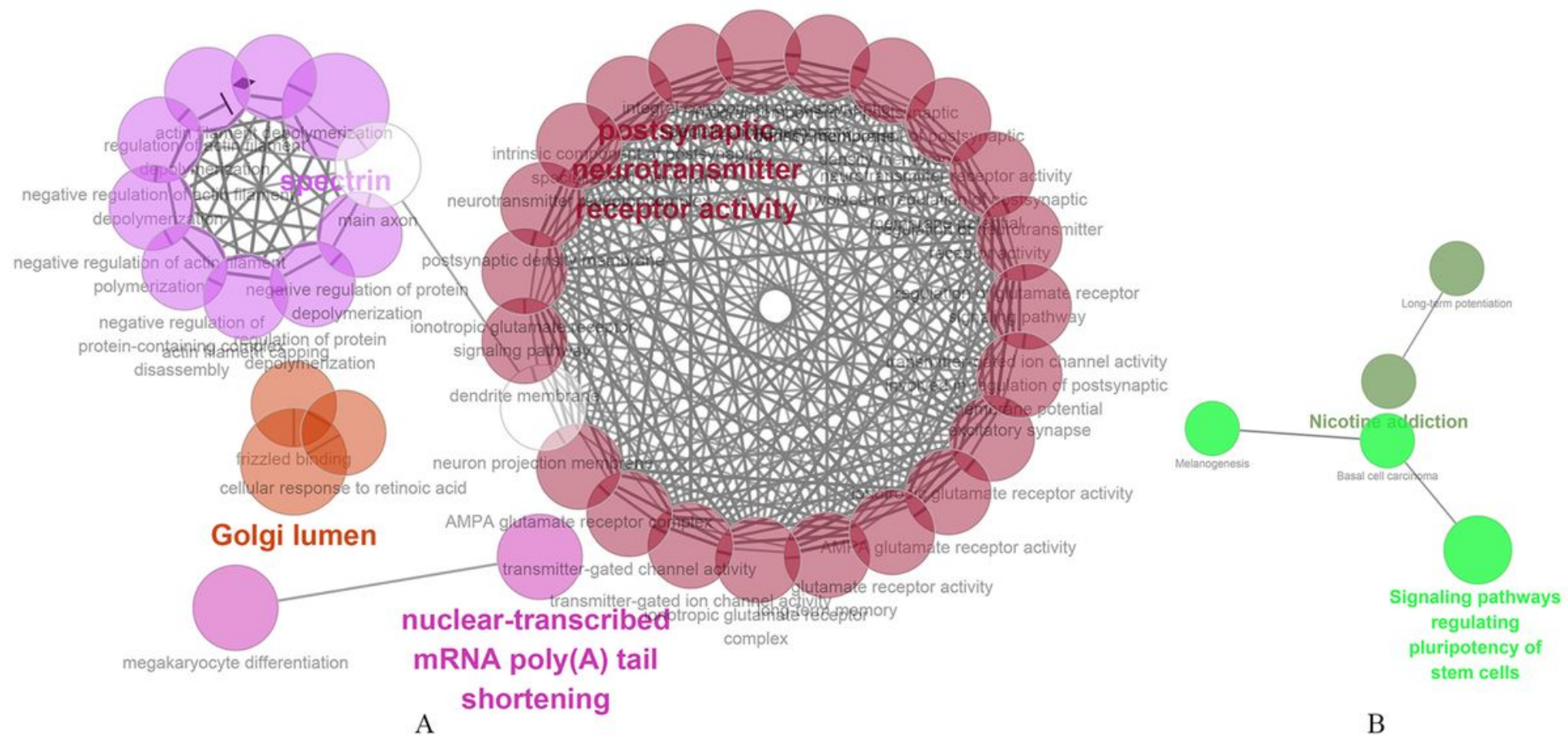

B

Figure 5

Enrichment results of GO and KEGG of 5 top modules. A: GO enrichment results ,each circle represents the biological process, cell component or molecular function of enrichment, with different levels of color enrichment. The larger the circle, the more genes are enriched in this Term. B: KEGG enrichment results, each circle represents the enriched signaling pathway, with different levels of color enrichment. The larger the circle, the more genes are enriched in this Term.

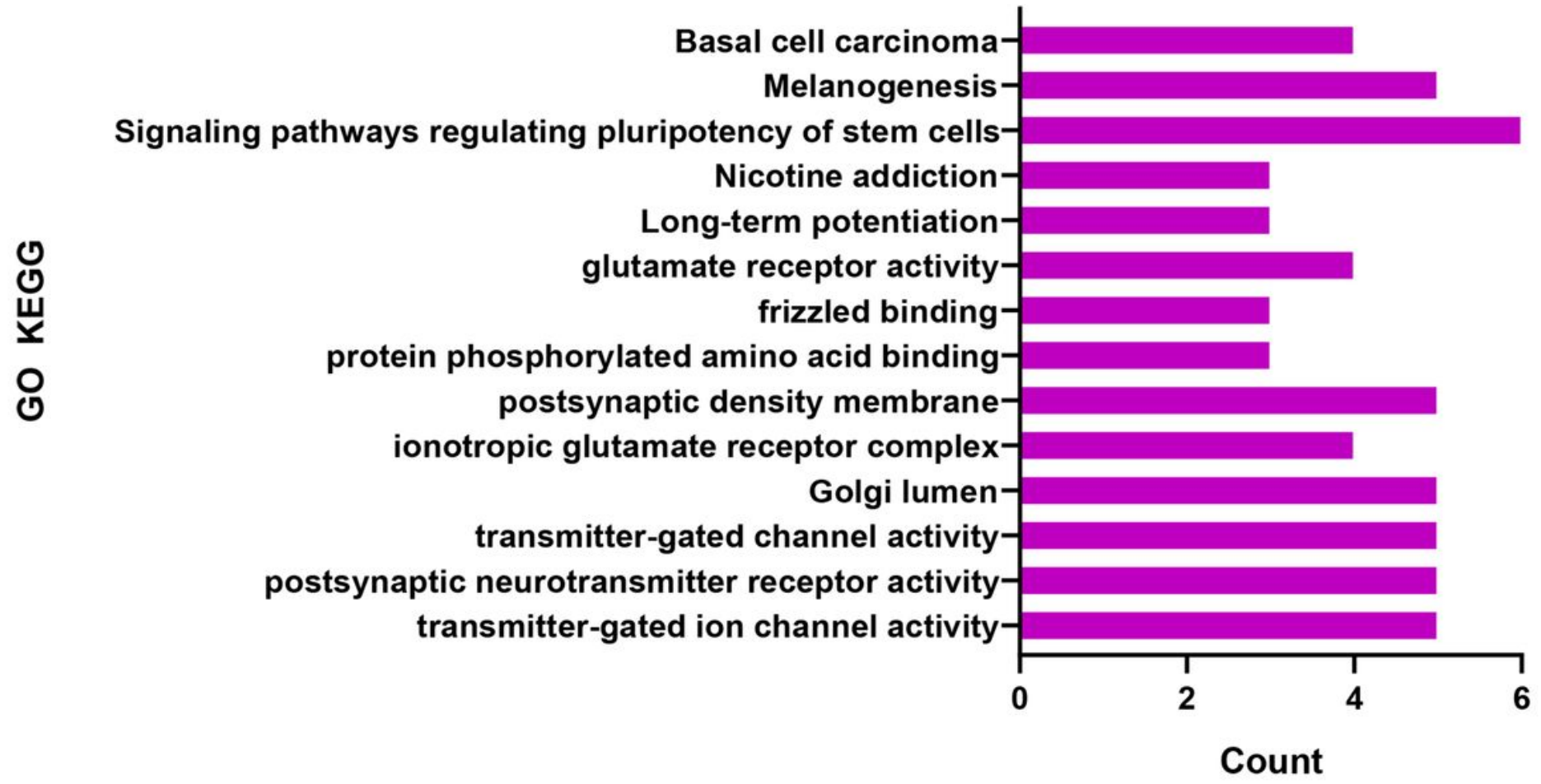

Figure 6 
The number of genes in each enrichment region of the top modules. abscissa: gene count, ordinate: results of Go and KEGG enrichment.

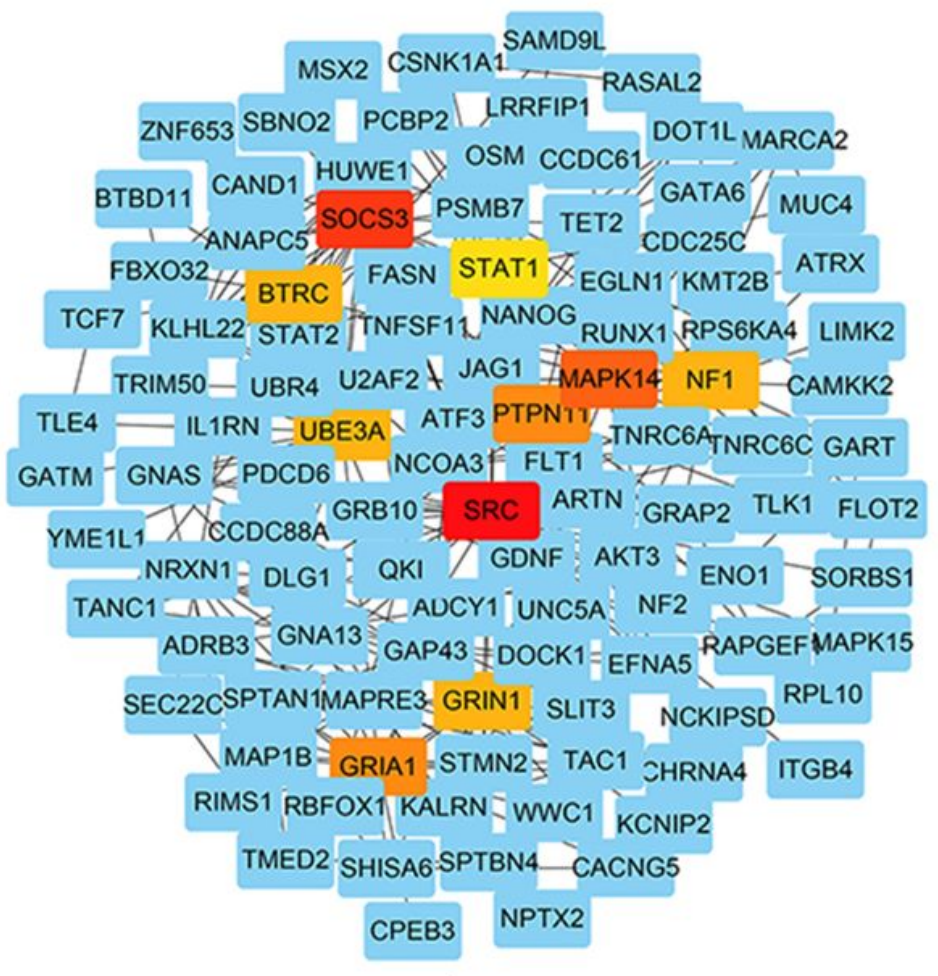

A

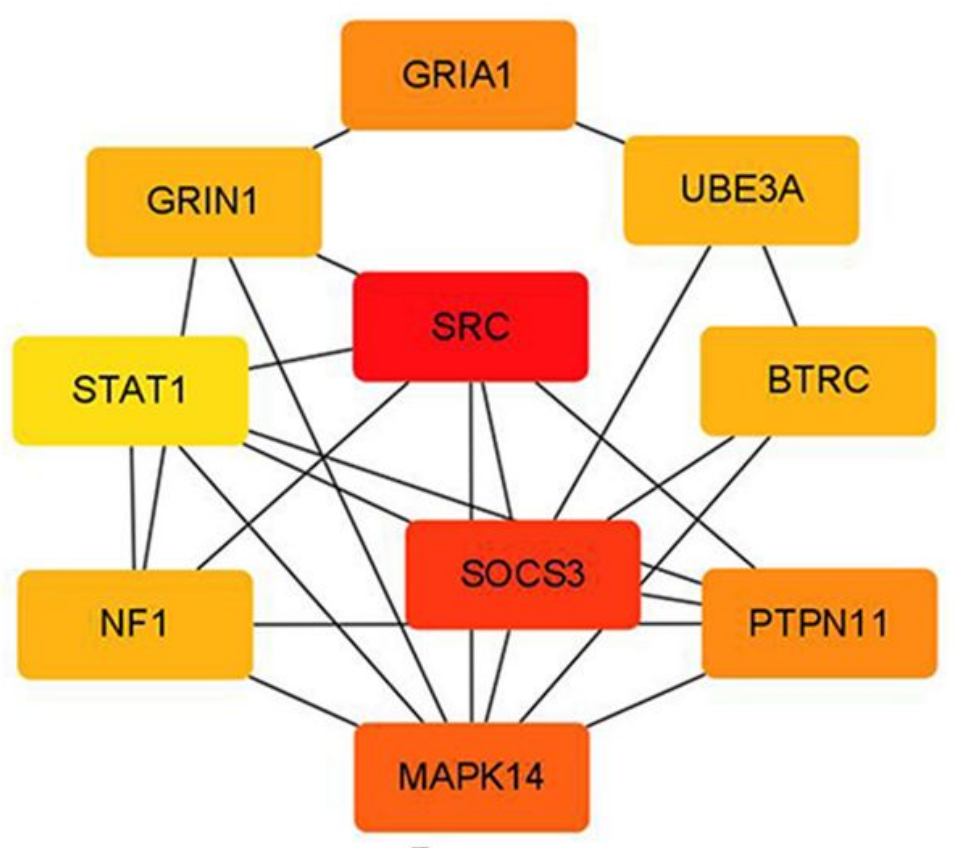

B

\section{Figure 7}

Hub gene screened from PPI. A:PPI shows the Hub gene. Squares represent differential genes, and the darker ones are Hub genes. B: Hub gene interaction diagram. Hub gene is derived from the Degree algorithm on Cytohubba, a plugin for Cytoscape. 


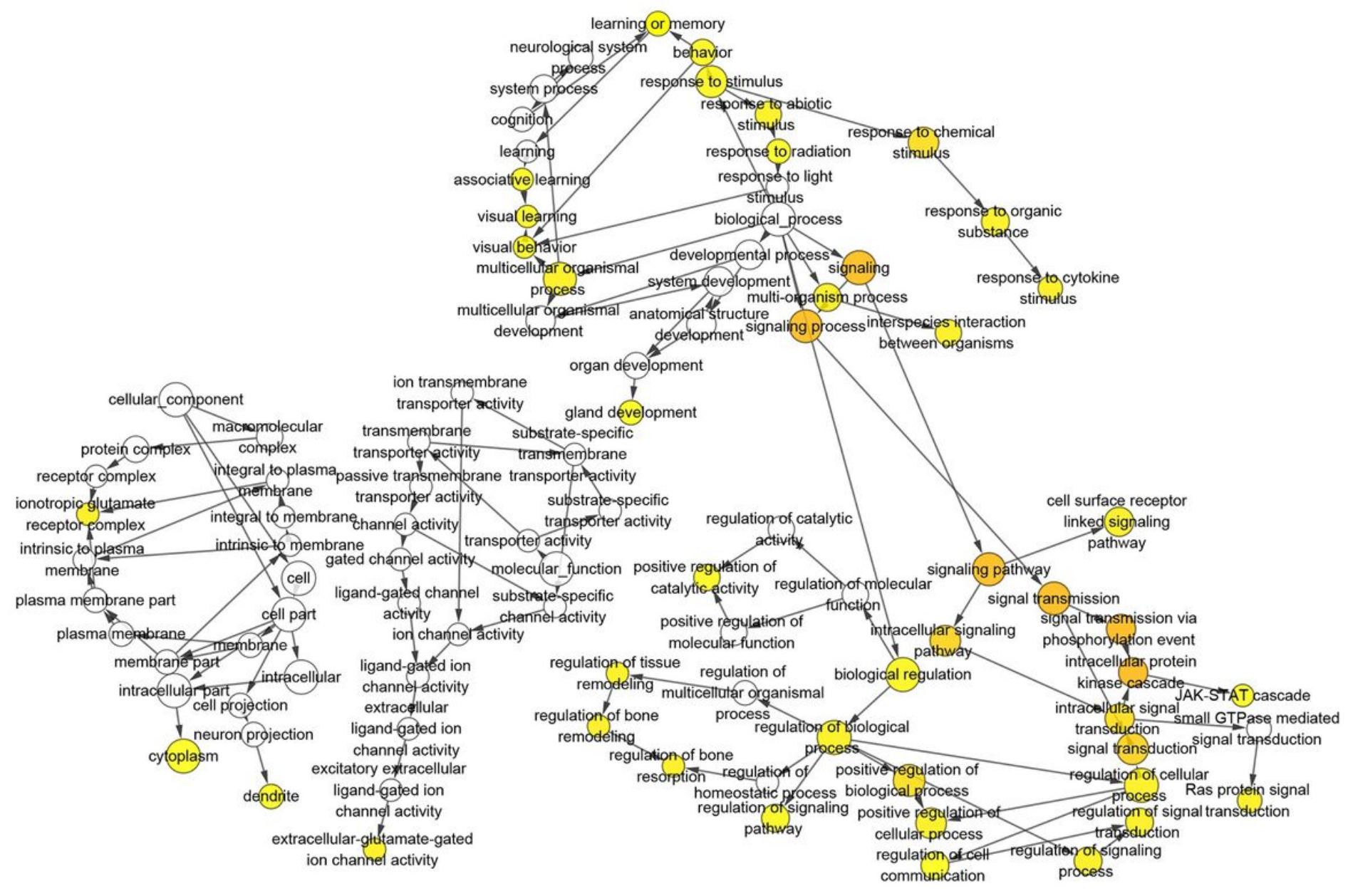

\section{Figure 8}

As a result of Hub gene GO enrichment, each circle represents a GO Term. The darker the color, the higher the significance, and the larger the circle, the more genes are enriched in this Term. 


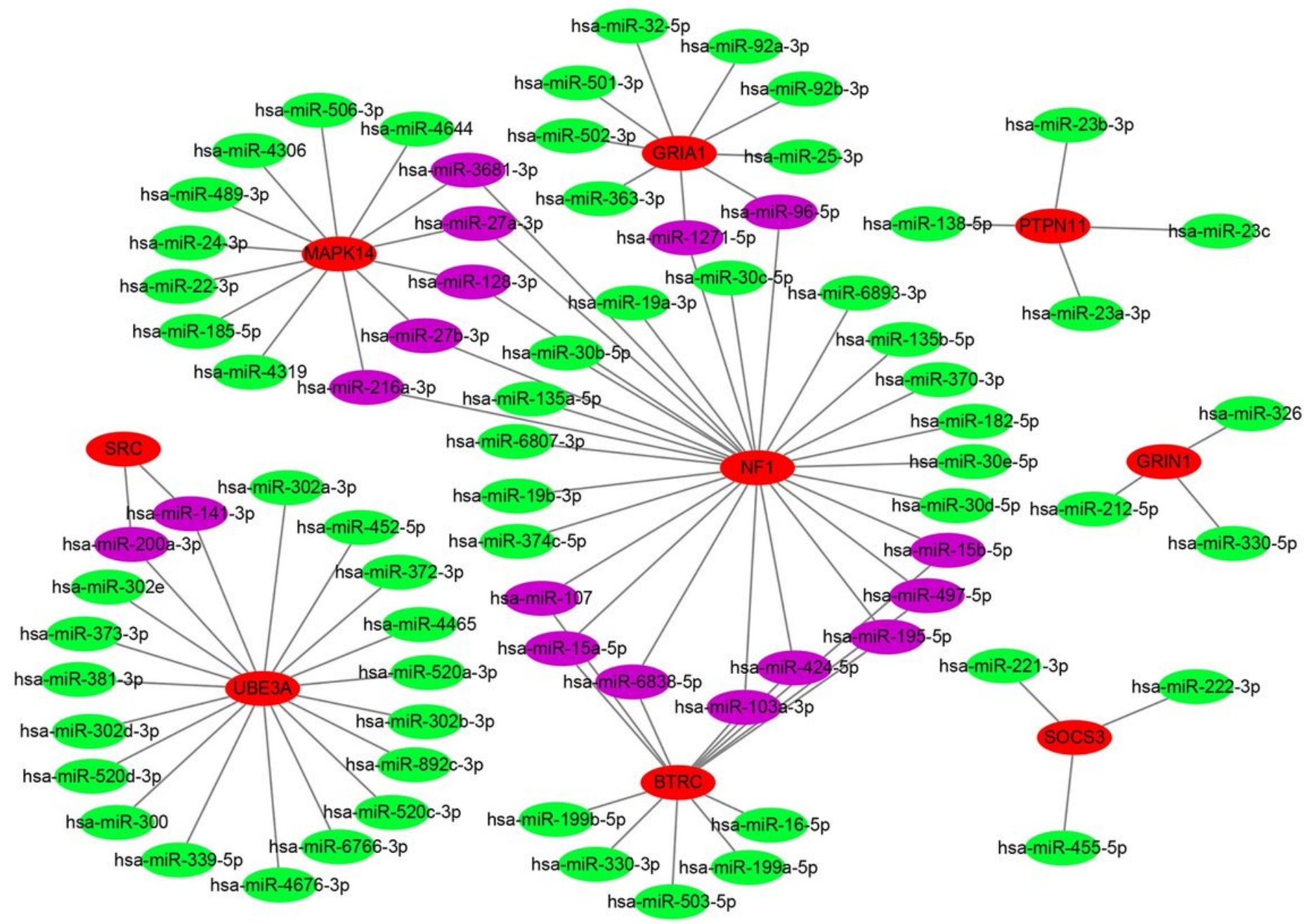

Figure 9

The interaction network between the Hub gene and its targeted miRNA. Genes are stained red, and the node size is adjusted according to the number of targeted miRNAs, miRNAs are stained green, and miRNAs that target two or more genes are purple. 